\title{
Iridium-Catalyzed Asymmetric a-Allylic Alkylation of Amides using Vinyl Azide as Amide Enolate Surrogate
}

\author{
Aditya Chakrabarty, and Santanu Mukherjee* \\ Department of Organic Chemistry, Indian Institute of Science, Bangalore - 560012, India \\ Supporting Information Placeholder
}

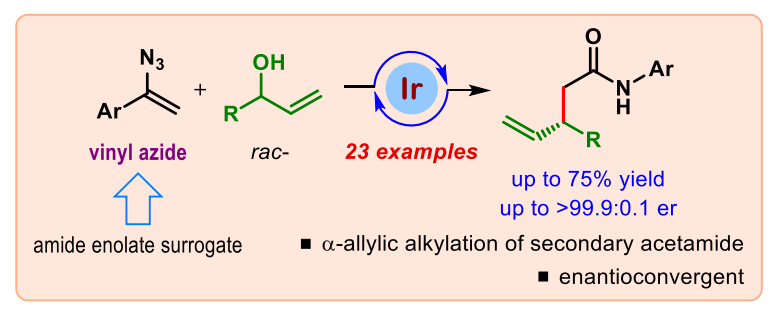

ABSTRACT: Among the unstabilized enolates used as nucleophile in iridium-catalyzed asymmetric allylic alkylation reactions, amide enolates are least explored. Vinyl azides are now employed as amide enolate surrogate for the first time in Ir-catalyzed asymmetric allylic alkylation with branched allylic alcohols as the allylic electrophile. Competing reaction pathways are suppressed through systematic tuning of steric and electronic properties of vinyl azide to effect $\alpha$-allylic alkylation of secondary acetamides with high atom-economy, exclusive branched selectivity and moderate to excellent enantioselectivity.

Notwithstanding the phenomenal developments in the field of iridium-catalyzed asymmetric allylic substitution (AAS) reactions in the past two decades, ${ }^{1}$ stabilized enolates remain among the most popular and privileged class of carbon nucleophiles. ${ }^{1,2}$ In contrast, asymmetric allylic alkylation (AAA) of unstabilized enolates are much less explored. In this context, the paucity of amide enolates in Ir-catalyzed AAA is particularly noteworthy.

Due to the reduced acidity of the $\alpha$-protons, ${ }^{3}$ generation of amide enolates requires strong base and such conditions are often incompatible with some of the AAA conditions (Scheme 1A). ${ }^{4}$ Consequently, efforts were mostly confined to easily enolizable oxazolones and thiazolones as precursors for highly substituted amides. ${ }^{5,6}$ In 2018, Hartwig et al. addressed the low acidity of amides through the use of a synergistic combination of iridium and copper catalysis, to develop a stereodivergent allylic alkylation of $\alpha$-azaaryl acetamides (Scheme 1B). ${ }^{7}$

However, enantioselective $\alpha$-allylic alkylation of $\alpha$-unsubstituted acetamides continued to elude the grasp of available synthetic tools. In 2019, Carreira and co-workers came up with an ingenious solution to this problem by using morpholine ketene aminal as the amide enolate equivalent to develop the first catalytic enantioselective formal allylic alkylation of $\alpha$-unsubstituted acetamides (Scheme 1B). ${ }^{8}$ Using branched allylic carbonates as the allylic electrophile, this reaction proceeds with kinetic resolution and gives rise to $\alpha$-allyl tertiary amides with excellent enantioselectivity.

In spite of these notable advances, enantioselective $\alpha$-allylic alkylation of primary and secondary acetamides with the generation of a $\beta$-stereogenic center is yet to be accomplished.

Scheme 1. Ir-Catalyzed Asymmetric $\alpha$-Allylic Alkylation (AAA) of Amides

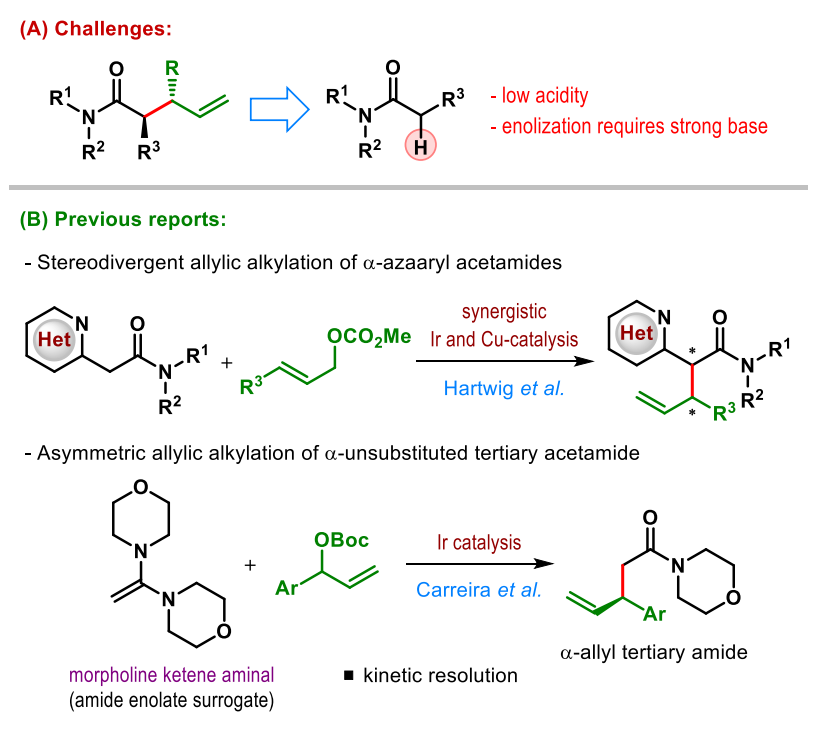

(C) This work: Asymmetric allylic alkylation of secondary acetamides

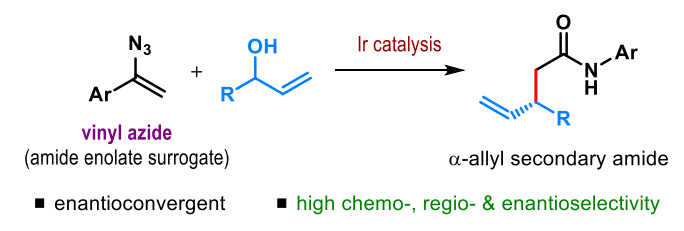


Vinyl azides, owing to their structural resemblance with enamine (vide infra), have recently emerged as an amide enolate surrogate since the pioneering work of Chiba and co-workers. ${ }^{9}$ While vinyl azides have been used for formal $\alpha$-functionalization of amides, ${ }^{10}$ their applications in enantioselective reaction are scarce. Only recently Terada et al. developed the first enantioselective reaction of vinyl azides for addition to $N$-acyl aldimines under chiral Brønsted acid catalysis. $^{11}$

With our interest in Ir-catalyzed AAS reactions, ${ }^{12}$ we envisaged that the reaction of vinyl azide with an in situ generated electrophilic $\pi$-allyl-Ir complex could lead to an overall $\alpha$-allylic alkylation of $\alpha$-unsubstituted secondary acetamides (Scheme 1C).

However, this catalytic strategy is associated with a number of challenges (Scheme 2). Nucleophilic addition of vinyl azide (A) to the more substituted terminal of an electrophilic $\pi$-allyl-Ir complex is expected to generate the branched iminodiazonium ion $\mathbf{B}$, which can form as a mixture of $(E)$ - and $(Z)$-isomers. Schmidt-type rearrangement of $(E)$-B through the 1,2-aryl migration followed by hydration of the resulting nitrilium ion $\mathbf{C}$ would then furnish the $\alpha$-functionalized acetamide D. We surmised that using branched allylic alcohol as the allylic electrophile in combination with a Lewis or Brønsted acid promoter would make this process highly atom-economic by providing the hydroxide necessary for the conversion of nitrilium ion $\mathbf{C}$ to the product amide D. A competitive pathway involving $(Z)$-B would favor 1,2-alkyl migration to form an isomeric nitrilium ion $\mathbf{C}^{\prime}$ and eventually to $N$-homoallylbenzamide derivative D'. Controlling the geometry of $\mathbf{B}$ is crucial to ensure the desired aryl migration. Another competitive pathway comprises of the hydrolysis of iminodiazonium ion $\mathbf{B}$ to the $\alpha$-allyl ketone $\mathbf{E}$. The suppression of the latter two pathways would be the key to the success of this strategy. In addition, decomposition of vinyl azide to the corresponding methyl ketone through protonation and hydrolysis is yet another hurdle to be surmounted.

Scheme 2. Asymmetric Allylic Alkylation (AAA) of Vinyl Azides: Catalytic Hypothesis and Challenges

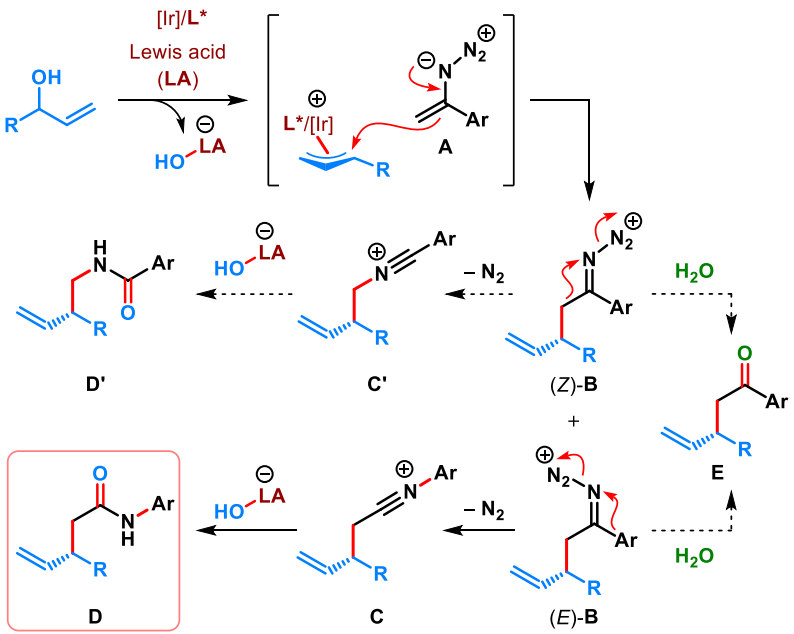

We herein report the results of our investigation, which successfully culminated in the first catalytic enantioselective allylic alkylation of vinyl azides en route to $\alpha$-allylic secondary amides. ${ }^{13}$
At the outset of this study, we tested the reactivity of 1phenyl vinyl azide 1a towards the $\pi$-allyl-Ir complex generated in situ from various combinations of allylic electrophiles and Ir-catalysts derived from either Feringa's phosphoramidite ligand ${ }^{14}\left(S_{a}, S, S\right)$-L1 or Carreira's (phosphoramidite,olefin)-ligand ${ }^{1 \mathrm{~b}}\left(S_{a}\right)$-L2 (Table 1$) .{ }^{15}$ The catalyst generated from $[\operatorname{Ir}(\mathrm{COD}) \mathrm{Cl}]_{2}$ and $\left(S_{a}, S, S\right)-\mathbf{L 1} \mathbf{1}^{16}$ was found to be completely inactive when used in combination with linear allylic carbonates. Similarly, no reaction took place when branched allylic carbonate was used under the influence of the catalyst generated from $[\operatorname{Ir}(\mathrm{COD}) \mathrm{Cl}]_{2}$ and $\left(S_{a}\right)$ L2. ${ }^{17}$

In contrast, the conversion of vinyl azide $1 \mathrm{a}$ was observed when the reaction was carried out using branched allylic alcohol 2a in the presence of only $3 \mathrm{~mol} \%[\operatorname{Ir}(\mathrm{COD}) \mathrm{Cl}]_{2}, 12$ mol \% $\left(S_{a}\right)$-L2 and 10 mol \% of Sc(OTf) 3 as the promoter in $\mathrm{THF}$ at $50{ }^{\circ} \mathrm{C}$. Unfortunately, the isolated product was found to be $\alpha$-allyl acetophenone $\mathbf{4}$ and no trace of the desired $\alpha$-allyl amide 3 aa was detected (Table 1, entry 1 ).

Table 1. Optimization of Reaction Parameters

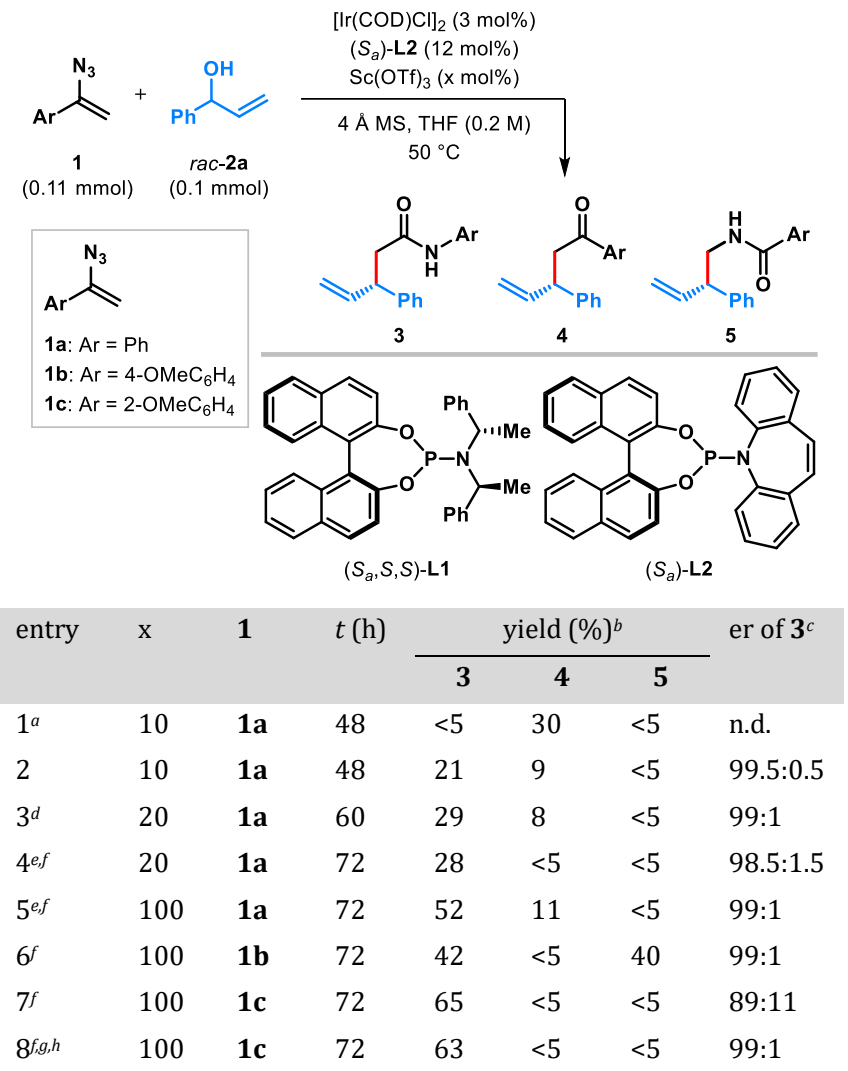

${ }^{a}$ Reaction without molecular sieves. ${ }^{b}$ Yields correspond to the isolated yield after chromatographic purification. ${ }^{c}$ Enantiomeric ratio (er) as determined by HPLC analysis on a chiral stationary phase. ${ }^{d}$ Using 1.5 equiv of $\mathbf{1 a}$. ${ }^{e}$ Reaction in 1,4-dioxane. fUsing 2.0 equiv of 2a. ${ }^{g}$ Reaction at $25^{\circ} \mathrm{C}$. ${ }^{\circ}$ Reaction on a $0.2 \mathrm{mmol}$ scale. MS $=$ molecular sieves. n.d. $=$ not determined

As anticipated (see Scheme 2), removal of moisture by adding $4 \AA$ MS suppressed the formation of ketone and resulted in substantial amount of the desired amide 3aa exclusively as the branched isomer with outstanding enantioselectivity (entry 2). Efforts in ameliorating the yield of 3aa by using other Lewis or Brønsted acidic promoters proved futile and $\mathrm{Sc}(\mathrm{OTf})_{3}$ remained the optimum. ${ }^{15}$ Use of 
a larger excess of vinyl azide in combination with $20 \mathrm{~mol} \%$ $\mathrm{Sc}(\mathrm{OTf})_{3}$ slightly improved the yield of 3aa (entry 3). Screening of solvents ${ }^{15}$ revealed 1,4-dioxane as the optimum (entry 4). The reaction with 2.0 equiv of allylic alcohol $2 \mathbf{a}$ and 100 mol \% of Sc(OTf) 3 in 1,4-dioxane led to $52 \%$ isolated yield of 3aa with 99:1 er (entry 5). Nevertheless, the modest yield of 3aa and the formation of a measurable amount of ketone 4 remained a cause of concern.

We reasoned that the use of a more electron-rich aryl substituent on the vinyl azide might favor the Schmidt rearrangement pathway over the undesired hydrolysis of the iminodiazonium intermediate (B in Scheme 2). Although the ketone formation was completely suppressed when the reaction was carried out with 1 -( $p$-methoxyphenyl) vinyl azide $\mathbf{1 b}$ in $\mathrm{THF}$ at $50^{\circ} \mathrm{C}$, the undesired alkyl migration was found to compete with aryl migration. The resulting products $\mathbf{3 b a}$ and $\mathbf{5}$ were isolated almost as a 1:1 mixture with excellent er of both $\mathbf{3 b a}$ and $\mathbf{5}$ (entry 6). As discussed above, the formation of $(E)$-iminodiazonium intermediate was deemed to be the key for a productive aryl migration. With the anticipation of increasing the steric bulk on the aryl ring to favor $(E)$-B over $(Z)$-B (see Scheme 2), while maintaining its electron-rich nature, we sought to apply 1-(o-methoxyphenyl) vinyl azide 1c as the amide enolate surrogate. We were pleased to note that the use of 1c eliminated both these undesired pathways and resulted in $\alpha$-allyl amide $\mathbf{3 c a}$ as the sole product, albeit with only 89:11 er (entry 7). Carrying out the reaction at $25^{\circ} \mathrm{C}$ restored the enantioselectivity to $99: 1$ er and 3ca was isolated in 63\% yield (entry 8). It must be noted that even though 2.0 equiv of racemic allylic alcohol (2a) was used in this reaction with respect to vinyl azide, no kinetic resolution was observed and the recovered 2 a remained racemic.

With the optimum reaction conditions (Table 1, entry 8) at hand, we tested the compatibility of this protocol with other branched allylic alcohols. As shown in Table 2A, allylic alcohols having electronically diverse aryl substituents were found to react smoothly with 1-(o-methoxyphenyl) vinyl azide 1c and furnished the corresponding $\alpha$-allyl $N$-arylacetamide derivatives in moderate to good yield with exquisite regio- (b vs l) as well as chemoselectivity. While the products derived from allylic alcohols containing electronrich aryl substituents were formed with moderate to good enantioselectivity (entries 2-4), allylic alcohols bearing electron-deficient aryl substituents at various positions fared extremely well and in most cases, the products were isolated with outstanding level of enantioselectivity. Only in the case of $m$-bromophenyl substituted allylic alcohol $\mathbf{2 m}$, the product ( $\mathbf{3} \mathbf{c m}$ ) was obtained with modest er (entry 13). Allylic alcohols bearing heteroaryl substituents such as dioxolane and thienyl (2t-u) also afforded the products with good to excellent enantioselectivity, albeit with somewhat less yield (entries 20-21). Please note that neither $\alpha$-allyl acetophenone nor $N$-homoallylbenzamide derivatives (E and $\mathbf{D}^{\prime}$, respectively in Scheme 2) were detected in any of these reaction. Unfortunately, no reaction was observed in the case of alkyl or alkenyl substituted allylic alcohols ${ }^{15}$ and marks a limitation of this protocol.

The scope of vinyl azide was also found to be very limited. Reaction with a number of 1-aryl vinyl azides apart from 1a-c were tested and no desired product was obtained in any of these cases. ${ }^{15}$ However, 1-phenyl vinyl azide 1a could be used with other branched allylic alcohols as shown for p-bromophenyl derivative $\mathbf{2 h}$ : Although the product $\mathbf{3 a h}$ was isolated with only 54\% yield, it was formed with 98:2 er (Table 2B). Enantioselectivity was further improved through a single recrystallization, which afforded diffraction quality crystals. Single-crystal X-ray diffraction analysis of 3 ah revealed its absolute configuration to be $(S)$ (CCDC 2020776, Table 2B). The configurations of the other products were tentatively assigned as the same by analogy.

Table 2. Scope Asymmetric Allylic Alkylation of Vinyl Azides $^{a}$

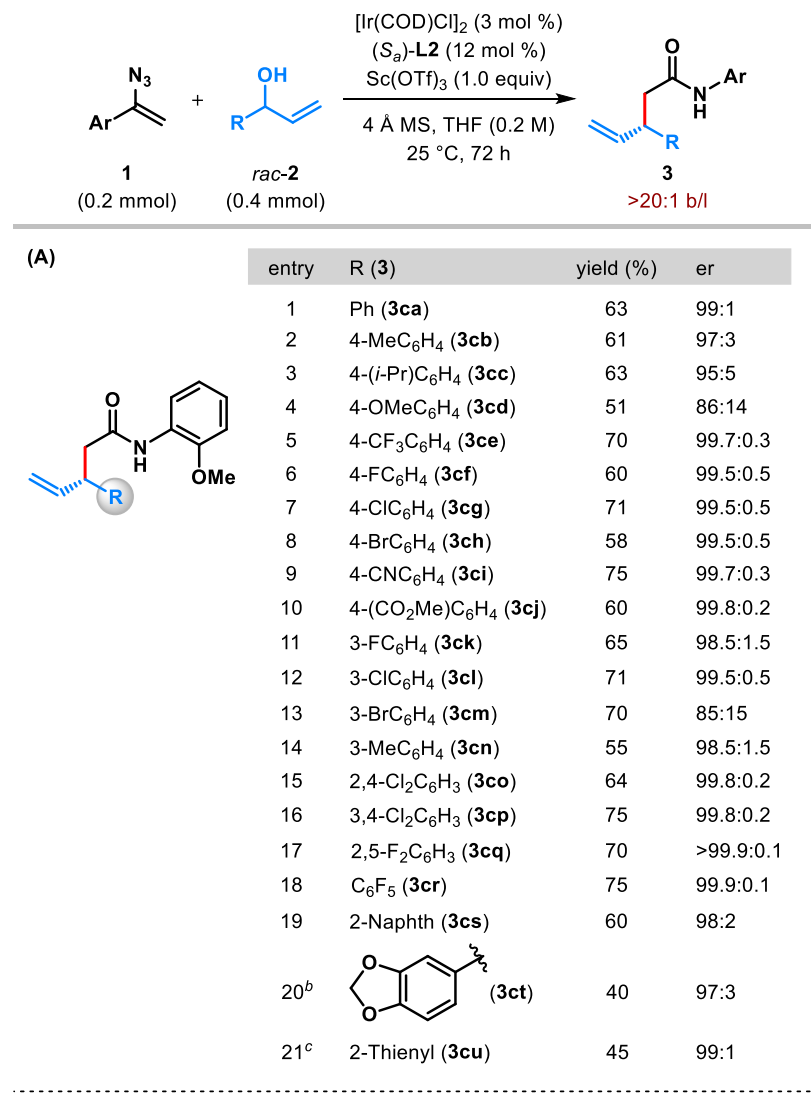

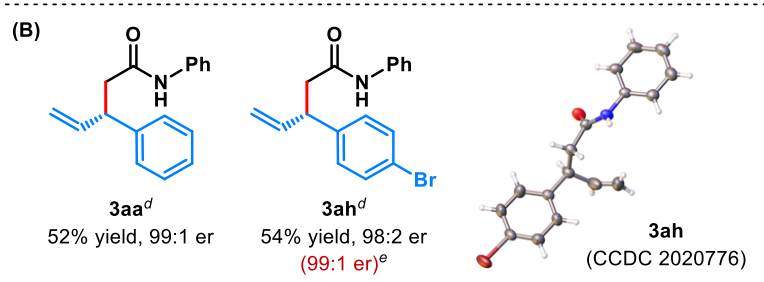

${ }^{a}$ Unless noted otherwise, the reaction conditions indicated above were followed. Yields refer to the isolated product after chromatographic purification. Enantiomeric ratios (er) were determined by HPLC analysis on a chiral stationary phase. ${ }^{b}$ Reaction with $50 \mathrm{~mol} \%$ $\mathrm{Sc}(\mathrm{OTf})_{3 .}{ }^{c}$ Reaction with $25 \mathrm{~mol} \% \mathrm{Sc}(\mathrm{OTf})_{3 .}{ }^{d}$ Reaction at $50^{\circ} \mathrm{C}$. $e$ The value in parenthesis indicate er of $\mathbf{3 a h}$ after a single recrystallization.

To verify the scalability of this protocol, the allylic alkylation reaction between vinyl azide $\mathbf{1 c}$ and allylic alcohol $\mathbf{2 l}$ was carried out on a $1.0 \mathrm{mmol}$ scale under our standard reaction conditions (Scheme 3 ). The corresponding $\alpha$-allyl $N$-arylacetamide $3 \mathbf{c l}$ was isolated in $66 \%$ yield with $>$ 99.5:0.5 er. The synthetic potential of the product was demonstrated through the conversion of the newly built 
functionalities. Amide of $\mathbf{3 c l}$ was converted to the corresponding thioamide 6 by refluxing with Lawesson's reagent in toluene. Protection of the secondary amide in $\mathbf{3 c l}$ with Boc enabled further functionalizations. For example, hydroboration of the terminal double bond of 7 with pinacolborane could be accomplished under Ir-catalysis ${ }^{18}$ and furnished the alkyl boronate 8 in $85 \%$ yield. Olefin cross-metathesis of $\mathbf{7}$ with methyl acrylate in the presence of Grubbs' $2^{\text {nd }}$ generation catalyst afforded the $\alpha, \beta$-unsaturated ester 9 in high yield.

Scheme 3. (A) Scale-up Synthesis and (B) Synthetic Elaboration of $\alpha$-Allyl $N$-Arylacetamide $3 \mathrm{cl}$
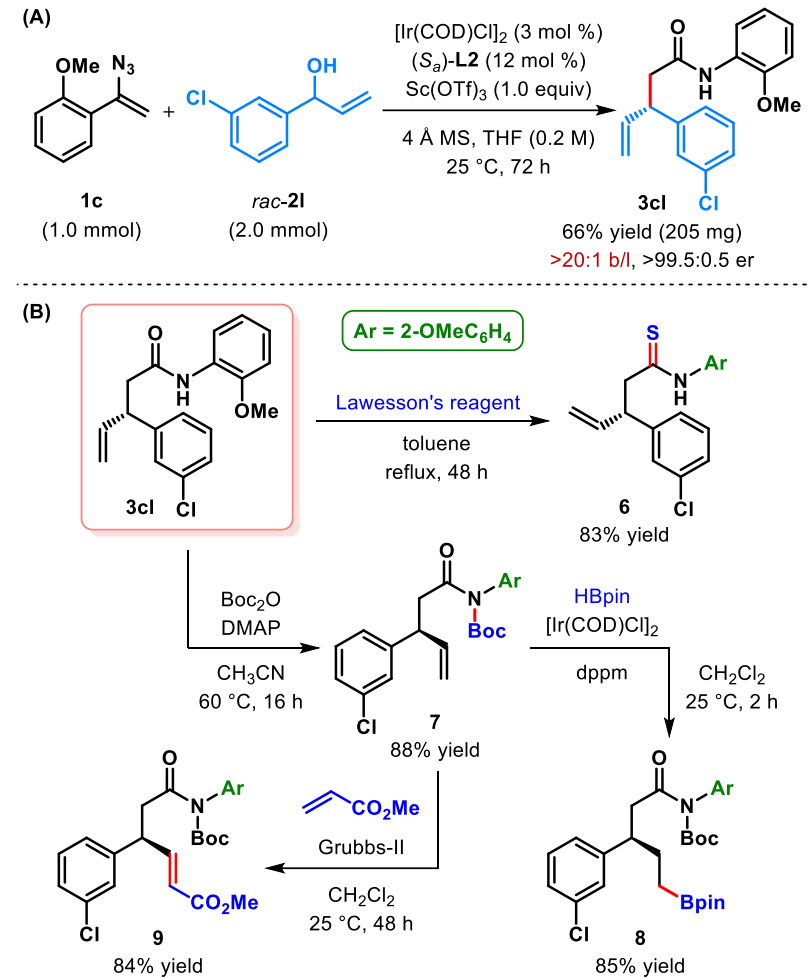

In summary, we have developed the first catalytic enantioselective allylic alkylation of vinyl azides as amide enolate surrogate. ${ }^{13}$ With $\mathrm{N}_{2}$ as the only byproduct, this highly atomeconomic transformation, catalyzed by $\operatorname{Ir}(\mathrm{I}) /($ phosphoramidite,olefin) complex, utilizes easy-to-prepare branched allylic alcohols as the allylic electrophile along with Sc(OTf) 3 as the Lewis acid promoter. Through systematic modulation of steric and electronic properties of vinyl azide, competing reaction pathways are circumvented and ultimately results in $\alpha$-allyl acetamides with exclusive branched selectivity and moderate to excellent enantioselectivity. Use of easily accessible allylic electrophiles and the reaction at ambient temperature are some of the practical advantages of our protocol. It also complements the use of morpholine ketene aminal as the amide enolate surrogate: $\alpha$-Allyl secondary amides are the products in this reaction, whereas the latter gives rise to $\alpha$-allyl tertiary amides.

\section{ASSOCIATED CONTENT}

\section{Supporting Information}

The Supporting Information is available free of charge via the Internet at http://pubs.acs.org
Experimental details and characterization data NMR spectra and HPLC chromatograms

\section{Accession Codes}

CCDC 2020776 contain the supplementary crystallographic data for this paper. These data can be obtained free of charge via www.ccdc.cam.ac.uk/data_request/cif, or by emailing data_request@ccdc.cam.ac.uk, or by contacting The Cambridge Crystallographic Data Centre, 12 Union Road, Cambridge CB2 1EZ, UK; fax: +44 1223336033 .

\section{AUTHOR INFORMATION}

\section{Corresponding Author}

*E-mail: sm@iisc.ac.in

ORCID

Aditya Chakrabarty: 0000-0003-3470-069X

Santanu Mukherjee: 0000-0001-9651-6228

\section{Notes}

The authors declare no competing financial interest.

\section{ACKNOWLEDGMENT}

Financial supports from the Science and Engineering Research Board (SERB) [Grant No. EMR/2016/005045] and the Council of Scientific and Industrial Research (CSIR) [Grant No. 02(0385)/19/EMR-II] are gratefully acknowledged. A.C. thanks Indian Institute of Science, Bangalore for a doctoral fellowship. High resolution mass spectra (HR-MS) were recorded on an equipment purchased from the Department of Science and Technology (DST)-FIST grant [Grant No. SR/FST/CS II040/2015]. We thank Mr. Rupak Saha (Department of Inorganic and Physical Chemistry, IISc, Bangalore) for his help with the X-ray structure analysis.

\section{REFERENCES}

(1) (a) Cheng, Q.; Tu, H.-F.; Zheng, C.; Qu, J.-P.; Helmchen, G.; You, S.L. Iridium-Catalyzed Asymmetric Allylic Substitution Reactions. Chem. Rev. 2019, 119, 1855-1969. (b) Rössler, S. L.; Petrone, D. A.; Carreira, E. M. Iridium-Catalyzed Asymmetric Synthesis of Functionally Rich Molecules Enabled by (Phosphoramidite,Olefin) Ligands. Acc. Chem. Res. 2019, 52, 2657-2672. (c) Shockley, S. E.; Hethcox, J. C.; Stoltz, B. M. Intermolecular Stereoselective Iridium-Catalyzed Allylic Alkylation: An Evolutionary Account. Synlett 2018, 29, 2481-2492. (d) Qu, J.; Helmchen, G. Applications of Iridium-Catalyzed Asymmetric Allylic Substitution Reactions in Target-Oriented Synthesis. Acc. Chem. Res. 2017, 50, 2539-2555. (e) Tosatti, P.; Nelson, A.; Marsden, S. P. Recent advances and applications of iridium-catalysed asymmetric allylic substitution. Org. Biomol. Chem. 2012, 10, 3147-3163. (f) Hartwig, J. F.; Stanley, L. M. Mechanistically Driven Development of Iridium Catalysts for Asymmetric Allylic Substitution. Acc. Chem. Res. 2010, 43, 1461-1475. (g) Helmchen, G.; Dahnz, A.; Dübon, P.; Schelwies, M.; Weihofen, R. IridiumCatalysed Asymmetric Allylic Substitutions. Chem. Commun. 2007, 675-691.

(2) For a review, see: Hethcox, J. C.; Shockley, S. E.; Stoltz, B. M. Iridium-Catalyzed Diastereo-, Enantio-, and Regioselective Allylic Alkylation with Prochiral Enolates. ACS Catal. 2016, 6, 6207-6213.

(3) Bordwell, F. G. Equilibrium Acidities in Dimethyl Sulfoxide Solution. Acc. Chem. Res. 1988, 21, 456-463.

(4) For selected exceptions, see: (a) Liu, X.-J.; Jin, S.; Zhang, W.-Y.; Liu, Q.-Q.; Zheng, C.; You, S.-L. Sequence-Dependent Stereodivergent Allylic Alkylation/Fluorination of Acyclic Ketones. Angew. Chem., Int. Ed. 2020, 59, 2039-2043. (b) Liu, X.-J.; You, S.-L. Enantioselective Iridium-Catalyzed Allylic Substitution with 2- 
Methylpyridines. Angew. Chem., Int. Ed. 2017, 56, 4002-4005. (c) Chen, X. J. W.; Hartwig, J. F. Iridium-Catalyzed Diastereoselective and Enantioselective Allylic Substitutions with Acyclic $\alpha$-Alkoxy Ketones. Angew. Chem., Int. Ed. 2016, 55, 5819-5823. (d) Liu, W.-B.; Okamoto, N.; Alexy, E. J.; Hong, A. Y.; Tran, K.; Stoltz, B. M. Enantioselective $\gamma$-Alkylation of $\alpha, \beta$-Unsaturated Malonates and Ketoesters by a Sequential Ir-Catalyzed Asymmetric Allylic Alkylation/Cope Rearrangement. J. Am. Chem. Soc. 2016, 138, 5234-5237. (e) Kanayama, T.; Yoshida, K.; Miyabe, H.; Takemoto, Y. Enantioand Diastereoselective Ir-Catalyzed Allylic Substitutions for Asymmetric Synthesis of Amino Acid Derivatives. Angew. Chem., Int. Ed. 2003, 42, 2054-2056.

(5) For selected examples, see: (a) Wang, T.; Yu, Z.; Hoon, D. L.; Huang, K.-W.; Lan, Y.; Lu, Y. Highly Enantioselective Construction of Tertiary Thioethers and Alcohols via Phosphine-Catalyzed Asymmetric $\gamma$-Addition Reactions of $5 \mathrm{H}$-Thiazol-4-ones and $5 \mathrm{H}$ Oxazol-4-ones: Scope and Mechanistic Understandings. Chem. Sci. 2015, 6, 4912-4922. (b) Chen, W.; Hartwig, J. F. Cation Control of Diastereoselectivity in Iridium-Catalyzed Allylic Substitutions. Formation of Enantioenriched Tertiary Alcohols and Thioethers by $\mathrm{Al}-$ lylation of 5H-Oxazol-4-ones and $5 \mathrm{H}$-Thiazol-4-ones. J. Am. Chem. Soc. 2014, 136, 377-382. (c) Trost, B. M.; Dogra, K.; Franzini, M. $5 H$ Oxazol-4-ones as Building Blocks for Asymmetric Synthesis of $\alpha$ Hydroxycarboxylic Acid Derivatives. J. Am. Chem. Soc. 2004, 126, 1944-1945.

(6) For selected indirect approaches, see: (a) Korch, K. M.; Eidamshaus, C.; Behenna, D. C.; Nam, S.; Horne, D.; Stoltz, B. M. Enantioselective Synthesis of $\alpha$-Secondary and $\alpha$-Tertiary Piperazin-2-ones and Piperazines by Catalytic Asymmetric Allylic Alkylation. Angew. Chem., Int. Ed. 2015, 54, 179-183. (b) Behenna, D. C.; Liu, Y.; Yurino, T.; Kim, J.; White, D. E.; Virgil, S. C.; Stoltz, B. M. Enantioselective Construction of Quaternary $N$-Heterocycles by Palladium-Catalysed Decarboxylative Allylic Alkylation of Lactams. Nat. Chem. 2012, 4, 130-133. (c) Zhang, K.; Peng, Q.; Hou, X.-L.; Wu, Y.-D. Highly Enantioselective Palladium-Catalyzed Alkylation of Acyclic Amides. Angew. Chem., Int. Ed. 2008, 47, 1741-1744. (d) Schelwies, M.; Dübon, P.; Helmchen, G. Enantioselective Modular Synthesis of 2,4-Disubstituted Cyclopentenones by Iridium-Catalyzed Allylic Alkylation. Angew. Chem., Int. Ed. 2006, 45, 2466-2469.

(7) Jiang, X.; Boehm, P.; Hartwig, J. F. Stereodivergent Allylation of Azaaryl Acetamides and Acetates by Synergistic Iridium and Copper Catalysis. J. Am. Chem. Soc. 2018, 140, 1239-1242.

(8) Sempere, Y.; Alfke, J. L.; Rössler, S. L.; Carreira, E. M. Morpholine Ketene Aminal as Amide Enolate Surrogate in Iridium-Catalyzed Asymmetric Allylic Alkylation. Angew. Chem., Int. Ed. 2019, 58, 9537-9541.

(9) (a) Zhang, F.-L.; Wang, Y.-F.; Lonca, G. H.; Zhu, X.; Chiba, S. Amide Synthesis by Nucleophilic Attack of Vinyl Azides. Angew. Chem., Int. Ed. 2014, 53, 4390-4394. For reviews, see: (b) Hayashi, H.; Kaga, A.; Chiba, S. Application of Vinyl Azides in Chemical Synthesis: A Recent Update. J. Org. Chem. 2017, 82, 11981-11989. (c) Fu, J.; Zanoni, G.; Anderson, E. A.; Bi, X. $\alpha$-Substituted Vinyl Azides: An Emerging Functionalized Alkene Chem. Soc. Rev. 2017, 46, 72087228.

(10) (a) Das, D. K.; Kannaujiya, V. K.; Sadhu, M. M.; Ray, S. K.; Singh, V. K. $\mathrm{BF}_{3} \cdot \mathrm{OEt}_{2}$-Catalyzed Vinyl Azide Addition to in Situ Generated N-Acyl Iminium Salts: Synthesis of 3-Oxoisoindoline-1-acetamides. J. Org. Chem. 2019, 84, 15865-15876. (b) Rasool, F.; Ahmed, A.; Hussain, N.; Yousuf, S. K.; Mukherjee, D. One-Pot Regioselective and Stereoselective Synthesis of C-Glycosyl Amides from Glycals Using
Vinyl Azides as Glycosyl Acceptors. Org. Lett. 2018, 20, 4036-4039. (c) Lin, C.; Shen, Y.; Huang, B.; Liu, Y.; Cui, S. Synthesis of Amides and Nitriles from Vinyl Azides and p-Quinone Methides. J. Org. Chem. 2017, 82, 3950-3956. (d) Zhang, Z.; Kumar, R. K.; Li, G.; Wu, D.; Bi, X. Synthesis of 4-Ynamides and Cyclization by the Vilsmeier Reagent to Dihydrofuran-2(3H)-ones. Org. Lett. 2015, 17, 61906193. (e) Zhang, F.-L.; Zhu, X.; Chiba, S. Tf 2 NH-Catalyzed Amide Synthesis from Vinyl Azides and Alcohols. Org. Lett. 2015, 17, 3138-3141.

(11) Nakanishi, T.; Kikuchi, J.; Kaga, A.; Chiba, S.; Terada, M. OnePot Synthesis of Enantioenriched $\beta$-Amino Secondary Amides via an Enantioselective [4+2] Cycloaddition Reaction of Vinyl Azides with N-Acyl Imines Catalyzed by a Chiral Brønsted Acid Chem.-Eur. J. 2020, 26, 8230-8234.

(12) (a) Sarkar, R.; Mukherjee, S. Enantioselective Direct Vinylogous Allylic Alkylation of 4-Methylquinolones under Iridium Catalysis. Org. Lett. 2019, 21, 5315-5320. (b) Sarkar, R.; Mitra, S.; Mukherjee, S. Iridium-Catalyzed Enantioselective Direct Vinylogous Allylic Alkylation of Coumarins. Chem. Sci. 2018, 9, 57675772.

(13) During the preparation of this manuscript, Cao et al. published a similar work using branched allylic carbonates as the allylic electrophile (first appeared as Just Accepted Manuscript on 14 July 2020): Han, M.; Yang, M.; Wu, R.; Li, Y.; Jia, T.; Gao, Y.; Ni, H.-L.; Hu, P.; Wang, B.-Q.; Cao, P. Highly Enantioselective Iridium-Catalyzed Coupling Reaction of Vinyl Azides and Racemic Allylic Carbonates. J. Am. Chem. Soc. 10.1021/jacs.0c01766.

(14) Teichert, J. F.; Feringa, B. L. Phosphoramidites: Privileged Ligands in Asymmetric Catalysis. Angew. Chem., Int. Ed. 2010, 49, 2486-2528.

(15) For details, see the Supporting Information.

(16) (a) Madrahimov, S. T.; Markovic, D.; Hartwig, J. F. The Allyl Intermediate in Regioselective and Enantioselective Iridium-Catalyzed Asymmetric Allylic Substitution Reactions. J. Am. Chem. Soc. 2009, 131, 7228-7229. (b) Markovic, D.; Hartwig, J. F. Resting State and Kinetic Studies on the Asymmetric Allylic Substitutions Catalyzed by Iridium-Phosphoramidite Complexes. J. Am. Chem. Soc. 2007, 129, 11680-11681. (c) Bartels, B.; García-Yebra, C.; Helmchen, G. Asymmetric IrI-Catalysed Allylic Alkylation of Monosubstituted Allylic Acetates with Phosphorus Amidites as Ligands Eur. J. Org. Chem. 2003, 1097-1103. (d) Ohmura, T.; Hartwig, J. F. Regioand Enantioselective Allylic Amination of Achiral Allylic Esters Catalyzed by an Iridium-Phosphoramidite Complex. J. Am. Chem. Soc. 2002, 124, 15164-15165.

(17) (a) Rössler, S. L.; Krautwald, S.; Carreira, E. M. Study of Intermediates in Iridium-(Phosphoramidite,Olefin)-Catalyzed Enantioselective Allylic Substitution. J. Am. Chem. Soc. 2017, 139, 36033606. (b) Defieber, C.; Ariger, M. A.; Moriel, P.; Carreira, E. M. Iridium-Catalyzed Synthesis of Primary Allylic Amines from Allylic Alcohols: Sulfamic Acid as Ammonia Equivalent. Angew. Chem., Int. Ed. 2007, 46, 3139-3143.

(18) Yamamoto, Y.; Fujikawa, R.; Umemoto, T.; Miyaura, N. IridiumCatalyzed Hydroboration of Alkenes with Pinacolborane. Tetrahedron 2004, 60, 10695-10700. 